\title{
Análisis del Síndrome de Edorexia y su relación con la actividad física en población universitaria
}

\section{Analysis of Edorexia Syndrome and their relationship with physical activity in universitary population}

Fecha de recepción: 18-05-2019

Fecha de aceptación: 10-12-2019
José Luis López-Morales

Universidad de Murcia

Francisco José Ortin Moreno

Universidad de Murcia

Enrique Javier Garcés de los Fayos Ruiz

\section{resumen/abstract:}

El deporte y los trastornos de la conducta alimentaria tienen una estrecha relación. En concreto, este artículo pretende profundizar en el Sindrome de Edorexia y su relación con el deporte. Los participantes fueron 89 estudiantes de la universidad de Murcia comprendidos entre 20 y 29 años, que cumplimentaron un autorregistro de actividad física y el Inventario de Edorexia. Nuestros resultados muestran como el Síndrome de Edorexia se relaciona con la práctica de la actividad física y el mecanismo de evitación. Estos datos se explican mediante la teoría del Síndrome de Edorexia y en sintonía con la práctica de deporte para conseguir un alivio de un malestar como, por ejemplo el miedo a engordar o la sensación de pérdida de control. Por tanto, el empleo de la actividad física como una herramienta para aumentar el autocontrol puede provocar el aumento del apetito y derivar a un Síndrome de Edorexia.

Sports and eating disorders are closely related. Specifically, this article intends to deepen in Edorexia Syndrome and your relationship with physical activity. The participants were 89 students from the University of Murcia between 20 and 29 years old completed a self-registration of the practice of physical activity and The Inventory of Edorexia. Our results show how the Edorexia Syndrome is related to practicing 3 or more times per week, this physical practice is particularly associated with the dimension of avoidance. These data are explained by the theoretical framework of the Edorexia Syndrome, consistent with the sport practice oriented towards the relief of distress, such as the fear of gaining weight or the sensation of loss of control. Hence, the practice of physical activity can be contemplated as a resource to increase self-control, which can lead in turn to an increased appetite and eventually to the Edorexia Syndrome.

\section{palabras clave/keywords:}

Actividad física, Edorexia, universitarios.

Physical activity, Edorexia, universitary.

\section{Introducción}

Los trastornos alimentarios no especificados son trastornos poco estudiados, aunque no implica que sean enfermedades menos graves que los trastornos clásicos. Por ejemplo, estudios recientes confirman que no existen diferencias entre los trastornos no especificados con la Bulimia Nerviosa (Turner, Bryant-Waugh, \& Peveler, 2010). En el presente estudio, se pretende estudiar más el sindroma de Edorexia y como este problema mental influye en la práctica de la actividad física. 
Se define el Síndrome de Edorexia como un problema psicológico que se caracteriza por una percepción del apetito constante, diario e incontrolable (López-Morales, 2017). Dos son las características principales de este síndrome, la primera son las demandas edoréxicas que hace referencia a una continuada percepción de apetito durante un breve periodo de tiempo o un aumento del apetito en comparación con el apetito de la mayoría de los individuos en situaciones similares. Estas demandas son incontrolables, automáticas (no son conductas planificadas), diarias y buscan un alivio inmediato. Según la teoría de déficit de bienestar psicológico (Blum, Liu, Shriner, Glod, 2011), el consumo de alimentos puede ser empleado para restablecer el equilibrio emocional del individuo. Por tanto, un individuo puede generar el deseo de comer excesivo con la finalidad de obtener un alivio temporal. Además, los individuos con Síndrome de Edorexia presentan episodios edoréxicos diferentes que pasamos a describir a nivel conductual (abuso de los alimentos o la evitación del consumo de los alimentos), a nivel emocional puede producirse culpabilidad o miedo y a nivel cognitivo se puede modificar los pensamientos y las creencias de los individuos para mantener el consumo de los alimentos.

El Síndrome de Edorexia destaca por la falta de control y el apetito constante y descontrolado. Sus dos tipos de conductas motoras reflejan ambas posibles respuestas, el consumo descontrolado o la evitación de la ingesta con la finalidad de evitar el descontrol. En coherencia con Fleur, Houshyar, Roy et al. (2005), el consumo de alimentos produce place. En este sentido, los episodios edoréxicos se producen principalmente con el propósito de consumir alimentos para aliviar una situación ansiogena. Además, la restricción de los alimentos durante la dieta como mecanismo de escape provoca un aumento de apetito (Massey y Hil, 2012) y un aumento del peso corporal como se ha observado en un estudio piloto anterior (López-Morales, 2013).

A diferencia de otros Trastornos alimentarios, los individuos con Síndrome de Edorexia presentan impulsividad, descontrol con la alimentación, abuso o restricción de alimentos. No obstante, no presentan purgas ni atracones (López-Morales, 2018), es decir, en su repertorio conductual no se incluye conductas clínicamente inadecuadas. Los individuos con Síndrome de Edorexia pueden presentar el miedo intenso a ganar peso y pueden recurrir a la restricción de alimentos, como la Anorexia Nerviosa. Sin embargo, estas restricciones serán relativas, se disminuye el consumo de alimentos, no son restricciones absolutas y no presentan una alteración de la imagen corporal. Además, la Edorexia presenta sensación de falta de control como en la Bulimia Nerviosa pero no presentan purgas y el abuso de los alimentos no está planificado, se caracteriza por un picoteo constante o un consumo ligeramente superior a la ingesta habitual. A diferencia del consumo normal de alimentos, el Síndrome de Edorexia son conductas prolongadas en el tiempo y provocan una alteración psicosocial y física.

En los diversos trastornos alimentarios se puede observar la práctica de compulsiva de actividad física hasta en un 85\% (Fietz, Touyz, y Hay, 2014). En concreto, el ejercicio compulsivo se ha definido como un impulso rígido y altamente impulsado para estar físicamente activo, en asociación con una incapacidad percibida para dejar de hacer ejercicio a pesar de que el individuo es consciente de las posibles consecuencias negativas (Taranis, Touyz, 
y Meyer, 2011). En relación a los trastornos alimentarios no especificados, presentan una actividad física compulsiva (Danielsen, Ro, Romild, y Bjornelv, 2016; Meyer et al., 2016; Sauchelli et al., 2016) al igual que los trastornos clásicos.

Las líneas de trabajo del Síndrome de Edorexia se centran en varios componentes que influyen en el comportamiento alimentario: la regulación emocional, el uso de conductas para escapa de un estímulo ansiogeno, la habituación del consumo excesivo de alimentos y el uso de la alimentación para resolver problemas. Por este motivo, el presente trabajo tiene el objetivo de analizar la relación del Síndrome de Edorexia con la práctica de actividad física.

Partiendo del objetivo se planten las siguientes hipótesis:

Los individuos que presenten Síndrome de Edorexia tenderán a realizar más actividad física. En consecuencia, con el modelo presentado, la práctica de la actividad física es consecuencia del miedo a engordar.

En los individuos con Sindrome de Edorexia, practicar actividad física estará relacionado con el mecanismo de evitación o escape del síndrome. Como indica la teoría del Síndrome de Edorexia, los mecanismos de evitación consisten en la evasión del miedo a engordar mediante el comportamiento alimentario o deportivo. Por ello, en aquellos individuos con este síndrome, su práctica deportiva estará relacionada con este mecanismo.

\section{Método}

\section{Participantes}

El reclutamiento muestral se realizó en la comunidad universitaria estableciendo como criterio de inclusión el rango de edad entre 20 y 29 años. Un total de 86 sujetos cumplieron este criterio, siendo la edad media 23.58 con una desviación típica de 2.45 . La mayoría de la muestra la constituían mujeres (65,3\% mujeres).

Con respecto al Síndrome de Edorexia, el 39.5\% presenta sintomatología edorexica. Estos participantes no presentan diferencias estadísticamente significativas en la edad $\left(\mathrm{T}_{78.907}=\right.$ $.819 \mathrm{p}=.416)$. Sin embargo, se observa diferencia estadísticamente significativa en el Î́ndice de Masa Corporal (IMC) debido a la fuerte relación que existe con la acumulación de grasa.

\section{Instrumentos y variables}

Cuestionario sociodemográfico. Se utilizó como instrumentos de evaluación un cuestionario sociodemográfico compuesto con preguntas acerca del género, edad, ocupación y residencia.

Variables antropométricas: IMC. Para el cálculo del Indice de Masa Corporal, se ha medido las variables antropométricas y pesado en una bioimpedancia. Después se ha calculado dividiendo el peso por la altura al cuadrado.

\section{Estado corporal}

Instrumento de evaluación del Síndrome de Edorexia (López-Morales, 2013). El cuestionario tipo test que consta de 47 items con cuatro respuestas diferentes cada uno. En ellas 
se evalúan conductas alimentarias y actitud hacía los alimentos. El cuestionario consta de 2 escalas, la escala general y escala especifica. La escala general tiene como finalidad evaluar las alteraciones psicosociales, así como las conductas patológicas propias del síndrome de Edorexia. La escala especifica se divide en 4 subescalas que evalúan las diferentes componentes del síndrome: evitación, dependencia, bienestar psicológico y emoción. Además, este cuestionario tiene una consistencia interna del .753 lo que le otorga fiabilidad para su uso en esta investigación. Para el diagnóstico del Síndrome de Edorexia se requiere una puntuación superior del percentil 70.

Actividad física. Se ha evaluado mediante un autorregistro de la frecuencia y duración de la actividad física. Posteriormente se clasificó a los sujetos en practicantes de actividad física o sedentarios en función de las horas practicadas. Se consideró que un individuo es sedentario practicaba menos de 3 días de ejercicio físico.

\section{Procedimientos}

Para la elaboración de este estudio, se inició el proceso de reclutamiento de la muestra de participantes, mediante una convocatoria a la comunidad universitaria de la Universidad de Murcia, a la que acudieron 92 individuos. Todos los participantes se agruparon en varias sesiones de recogida de muestras. Al principio de cada sesión, firmó el consentimiento informado de la recogida de datos para su uso en la investigación y se administró el cuestionario sociodemográfico y el cuestionario de evaluación del Síndrome de Edorexia en una misma sesión.

Una vez concluida la captación de datos, 6 sujetos se descartaron del estudio en aplicación de los criterios de exclusión: realizar actividad física vigorosa, definida como una actividad intensa que se realiza todos los días y durante varias horas. Por tanto, la muestra final se compuso de 86 participantes.

\section{Análisis estadístico}

Para la descripción de la muestra, actividad física, sedentarismo y Edorexia se empleó un análisis descriptivo con medias y desviación típica. Para la comparación de la actividad física y el sedentarismo con el Síndrome de Edorexia entre grupos edoréxicos y no edoréxicos se ha utilizado la prueba chi-cuadrado. Para la comparación de medias subescalas del Inventario del Síndrome de Edorexia se ha utilizado la prueba T de Student, previa verificación de la homogeneidad de variables mediante la prueba de Levene. Como medida del tamaño del efecto, se añade la d de Cohen para la comparación de medias. Todos los cálculos se han realizado con el programa estadístico SPSS (v.20)

\section{Resultados}

En referencia a la práctica de actividad física, el 51.2\% de la muestra no practica ninguna actividad física en comparación con el $27.9 \%$ que practica 3 o más días a la semana. Siendo el resto, actividad física ocasional (por ejemplo, algún día a la semana) o muy ocasional (por ejemplo, un o dos veces al mes), el 15.1\% y $5.8 \%$ respectivamente.

En el análisis de la actividad física entre el grupo edoréxicos y no edoréxicos, se analizó 
Tabla 1.- Diferencias en la práctica de actividad física y la presencia o ausencia del Síndrome de Edorexia

\begin{tabular}{lcccc}
\hline & No Ed & SíEd & $c^{2}$ & P \\
\hline No practica nada de actividad física & & & & \\
Practica ocasionalmente actividad física & $77.3 \%$ & $22.7 \%$ & 8.087 & .004 \\
Practica algún día a la semana actividad física & $60 \%$ & $40 \%$ & .038 & .846 \\
Practica más de 3 días a la semana actividad física & $75 \%$ & $25 \%$ & .563 & .453 \\
\hline
\end{tabular}

Nota: No Ed =Ausencia del Síndrome de Edorexia. Sí Ed = presencia del síndrome de edorexia.

Tabla 2.- Diferencias en las escalas del Inventario del Síndrome de Edorexia según la práctica de la actividad física

\begin{tabular}{lccccccc}
\hline & \multicolumn{2}{c}{ Sedentario } & \multicolumn{2}{c}{ Activo } & $\mathrm{t}$ & $\mathrm{pp}$ & $\mathrm{d}$ \\
\cline { 3 - 7 } & Media & DT & Media & DT & & & \\
\hline Escala General & 43.97 & 7.05 & 47.71 & 8.56 & -2.077 & .041 & .49 \\
D. B. F. & 9.08 & 2.92 & 9.12 & 3.04 & -.061 & .95 & .02 \\
Emocional & 8.84 & 2.69 & 9.83 & 2.43 & -1.578 & .118 & .37 \\
Evitación & 9.19 & 2.52 & 11.50 & 2.21 & -3.926 & .000 & .93 \\
Dependencia & 10.13 & 2.36 & 8.25 & 1.75 & .159 & .874 & -.04 \\
\hline
\end{tabular}

Nota: $\mathrm{DT}=$ Desviación típica. $\mathrm{t}=\mathrm{T}$ de Student. $\mathrm{d}=$ tamaño de efecto de Cohen. $\mathrm{p}=$ significación estadistica . D.B.F.= Déficit de bienestar psicológico.

n grupo alta intención: 223-226; $\mathrm{n}$ grupo baja intención: 28 ; en cursiva $\mathrm{CC}$ negativa y creencia de control facilitador

según su frecuencia. Entre los individuos que practican más de 3 días a la semana, el 66.7\% son edoréxicos, en comparación con el $33.3 \%$ de los individuos no edoréxicos. Esta diferencia es estadísticamente significativa $\left(\chi_{1}^{2}=12.364 ; p=, 000\right)$. Además, el $22.7 \%$ de los individuos que no practican actividad física son edoréxicos frente al $77.3 \%$ que tienen relación con el Síndrome de Edorexia $\left(\chi_{1}^{2}=8,087 ; \mathrm{p}=, 004\right)$. Como se observa en la tabla 1, el resto de las frecuencias, realizar actividad física algún día y ocasional no presentan diferencias estadísticamente significativas.

En un análisis más detallado del Síndrome de Edorexia, se compara los componentes del Síndrome de Edorexia con la práctica de 3 o más días de actividad física. En este análisis, en la Tabla 2 se destaca la dimensión evitación con una diferencia estadísticamente significativa $\left(\mathrm{t}_{84}=-3.926 ; \mathrm{p}=.000\right)$ y un tamaño de efecto muy alto según los criterios Cohen (1988). Además, siguiendo los mismos criterios se puede observar con un tamaño de efecto bajo en la escala general y la dimensión emocional. 


\section{Discusión}

Este estudio pretende profundizar en la relación de la actividad física con el Síndrome de Edorexia. La prevalencia del Síndrome de Edorexia en jóvenes universitarios es de 39.5\%, siendo muy elevada en comparación con los datos internacionales de la Trastornos de la Conducta Alimentaria clásicos (Ghaderi y Scott, 2001). Sin embargo, estos datos guardan relación con la tasa de sobrepeso española en jóvenes (Gutiérrez-Fisac et al., 2012). Por este motivo, el Síndrome de Edorexia puede explicar el aumento incontrolable de la obesidad española en sintonía con la falta de control de estas personas (Demos, Heatherton y Kelley, 2012).

En referencia con la actividad física, los edoréxicos practicaron actividad física con mayor frecuencia en comparación el grupo control. Estos datos confirman nuestra hipótesis y guardan relación con estudios anteriores que relacionan los trastornos alimentarios no especificados con la practica compulsiva de actividad física (Danielsen et al., 2016; Meyer et al., 2016; Sauchelli et al., 2016). La diferencia entre los individuos con edorexia y el grupo control parece indicar que el ejercicio se puede emplear como una herramienta para conseguir una finalidad como, por ejemplo, compensar la ingesta de alimentos o aliviar el malestar después de comer en exceso y así conseguir un alivio de sus emociones. El uso de actividad física como una técnica para aliviar síntomas no es nuevo, se emplea en diabetes (van Laake-Geelen, Smeets, Quadflieg, Kleijnen, y Verbunt, 2019), fibromialgia (Bonnabesse et al., 2019) e incluso en los trastornos alimentarios (Danielsen, Ro, y Bjornelv, 2018).

Al efecto de profundizar en el uso de la actividad física, se realiza un análisis del uso de la actividad física según los componentes del Síndrome de Edorexia. En este análisis, se observa una tendencia con el mecanismo de evitación. Este mecanismo consiste en la tendencia de evitar la sensación de pérdida de control y el consumo de alimentos. Los datos obtenidos confirman la hipótesis planteada y sugiere que la actividad física se puede emplear para continuar con el consumo excesivo de alimentos y, en consecuencia, para fines pocos saludables (Díaz, González y Dosil, 1998). Desde esta perspectiva, los individuos que presentan el mecanismo de escape para obtener el alivio de su malestar mediante la práctica de actividad física. Existen diferentes estudios que han relacionado la actividad física con una reducción de la sintomatología en otros trastornos como en la sintomatología depresiva (Moraska y Fleshner, 2001). Del mismo modo, la práctica de actividad física puede provocar un descenso del malestar y aliviar algunos síntomas, aunque en este caso se produce un efecto paradójico manteniendo la enfermedad.

Siguiendo esta línea y en coherencia con Eliot, Forker y Robertson (1976) se puede concluir que la actividad física puede mejorar la calidad de vida de los individuos reduciendo su malestar psicosocial. No obstante, su empleo como una herramienta para aumentar el autocontrol se puede comprender como una estrategia de afrontamiento centrada en las emociones y, a su vez, este tipo de afrontamiento se relaciona con un aumento del consumo de alimentos (Spoor, Bekker, Van Strien y van Heck, 2007), es decir la realización de deporte puede provocar un aumento del apetito (Edorexia) debido a su empleo como una herramienta de escape. 
En conclusión, la actividad física es una herramienta muy útil para el tratamiento de los trastornos alimentarios y mentales. Sin embargo, el empleo de la actividad física como una herramienta de evasión o su empleo abusivo o impulsivo podría estar relacionado con el Síndrome de Edorexia. En este síndrome, el uso de la actividad física se utiliza como una técnica que permite la evitación o el escape del malestar o estimulo ansiogeno y, por ello, en la prevención de los trastornos alimentarios en jóvenes universitarios se debe de incluir el empleo de la actividad física adecuada y la evitación del deporte como herramienta de alivio.

No obstante, este trabajo puede presentar limitaciones que se podrían mejorar mediante una muestra más amplia, el uso de un cuestionario estandarizado para la medición de la actividad física. En este sentido, futuro estudios deberán de profundizar en la relación de la relación de la actividad física con el Síndrome de Edorexia, en concreto con las consecuencias relacionadas con el mantenimiento de la actividad física impulsiva.

\section{Referencias}

Blum, K., Liu, Y., Shriner, R., y Gold, M.S. (2011). Reward circuitry dopaminergic activation regulates food and drug craving behavior. Current Pharmaceutical Design, 17 (12), 1158- 1167.

Bonnabesse, A. L. F., Cabon, M., L'Heveder, G., Kermarrec, A., Quinio, B., Woda, A., ... Bodere, C. (2019). Impact of a specific training programme on the neuromodulation of pain in female patient with fibromyalgia (DouFiSport): A 24-month, controlled, randomised, double-blind protocol. Bmj Open, 9(1), e023742.

Danielsen, M., Ro, O., y Bjornelv, S. (2018). How to integrate physical activity and exercise approaches into inpatient treatment for eating disorders: Fifteen years of clinical experience and research. Journal of Eating Disorders, 6, 34-43.

Danielsen, M., Ro, O., Romild, U., y Bjornelv, S. (2016). Impact of female adult eating disorder inpatients' attitudes to compulsive exercise on outcome at discharge and follow-up. Journal of Eating Disorders, 4, 7-17.

Demos, K.E., Heatherton, T.F., y Kelley, W.M. (2012). Individual differences in nucleus accumbens activity to food and sexual images predict weight gain and sexual behavior. The Journal of Neuroscience, 32 (16), $5549-5552$.

Diaz, O., González, O. y Dosil, J. (1998). Trastornos de alimentación. En VVAA, Materiales para la formación: Guía para padres y profesores. Madrid : CONCAPA.

Eliot, R. S., Forker, A. D., y Robertson, R. J. (1976). Aerobic exercise as a therapeutic modality in the relief of stress. Advances in Cardiology, 18, 231-42.

Fietz, M., Touyz, S., y Hay, P. (2014). A risk profile of compulsive exercise in adolescents with an eating disorder: A systematic review. Advances in Eating Disorders, 2(3), 241-263.

Fleur, S.E., Houshyar, H., Roy, M. y Dallman, M.F. (2005). Choice of lard, but not total lard calories, damps adrenocorticotropin responses to restraint. Endocrinology, 146, 2193-2199.

Ghaderi, A., y Scott, B. (2001). Prevalence, incidence and prospective risk factors for eating disorders. Acta Psychiatrica Scandinavica, 104(2), 122-130.

Gutiérrez-Fisac, J. L., Guallar-Castillón, P., León-Muñoz, L. M., Graciani, A., Banegas, J. R. y RodríguezArtalejo, F. (2012). Prevalence of general and abdominal obesity in the adult population of Spain, 2008-2010: the ENRICA study. Obesity Reviews: An Official Journal of the International Association for the Study of Obesity, 13(4), 388-392. 
López-Morales, J. L. (2018). Análisis del comportamiento alimentario y sus factores psicológicos en población universitaria no obesa. Anales de Psicología, 34(1), 1.

López-Morales, J. L. (2017) Síndrome de Edorexia: evaluación y correlatos (tesis doctoral). Universidad de Murcia.

Massey, A. y Hill, A. J. (2012). Dieting and food craving. A descriptive, quasi-prospective study. Appetite, 58 (3), 781-758.

Meyer, C., Plateau, C. R., Taranis, L., Brewin, N., Wales, J., y Arcelus, J. (2016). The Compulsive Exercise Test: Confirmatory factor analysis and links with eating psychopathology among women with clinical eating disorders. Journal of Eating Disorders, 4.

Moraska, A., y Fleshner, M. (2001). Voluntary physical activity prevents stress-induced behavioral depression and anti-KLH antibody suppression. American Journal of Physiology-Regulatory Integrative and Comparative Physiology, 281(2), R484-R489.

Sauchelli, S., Arcelus, J., Granero, R., Jimenez-Murcia, S., Aguera, Z., Del Pino-Gutierrez, A., y Fernandez-Aranda, F. (2016). Dimensions of Compulsive Exercise across Eating Disorder Diagnostic Subtypes and the Validation of the Spanish Version of the Compulsive Exercise Test. Frontiers in Psychology, 7, 1852.

Spoor, S. T. P., Bekker, M. H., Van Strien, T. y Van Heck, G. L. (2007). Relations between negative affect, coping, and emotional eating. Appetite, 48 (3), 368-376.

Taranis, L., Touyz, S., y Meyer, C. (2011). Disordered eating and exercise: Development and preliminary validation of the compulsive exercise test (CET). European Eating Disorders Review: The Journal of the Eating Disorders Association, 19(3), 256-268.

Turner, H., Bryant-Waugh, R., y Peveler, R. (2010). The clinical features of EDNOS: Relationship to mood, health status and general functioning. Eating Behaviors, 11(2), 127-130.

Van Laake-Geelen, C. C. M., Smeets, R. J. E. M., Quadflieg, S. P. A. B., Kleijnen, J., y Verbunt, J. A. (2019). The effect of exercise therapy combined with psychological therapy on physical activity and quality of life in patients with painful diabetic neuropathy: A systematic review. Scandinavian Journal of Pain, 19(3), 433-439. 